\title{
The effect of bilateral stimulation during sequential alternation of unilateral amygdaloid stimulation
}

\author{
JOHN GAITO \\ York University, Downsview, Ontario, Canada MSJ 1P3
}

\begin{abstract}
In two experiments, rats were subjected to a sequence of stimulations alternating from one amygdala to the contralateral one. Each phase of stimulation was for six convulsions prior to alternation to the other side. Rats In Experiment 1 had a series of six or seven phases of alternation, followed by five phases of bilateral stimulation, and concluded with seven phases of unilateral stimulation. In Experiment 2, rats had five phases of bilateral stimulation prior to seven phases of unilateral stimulation. The bilateral phases produced a modest disruption of the oscillation tendency when it was interspersed between two series of unilateral stimulation phases, more so in the criterion measure than in latency data. The effect was less on the oscillation tendency when bilateral stimulation preceded unilateral stimulation phases.
\end{abstract}

If an animal is stimulated unilaterally by low-intensity electrical current at periodic intervals, its behavior changes in an orderly fashion from no response to automatic behaviors (e.g., chewing, eye closure), and finally to clonic convulsions (CC). This event has been called the kindling effect (Goddard, McIntyre, \& Leech, 1969).

If one stimulates the amygdala (primary site) to a criterion (e.g., six CC), using $24 \mathrm{~h}$ as the intertrial interval, and then kindles the contralateral amygdala (secondary site), the criterion is reached within a fewer number of trials, but increased latency to convulsion results. Thus, both positive and negative transfer occurs (Goddard, McIntyre, \& Leech, 1969; McIntyre \& Goddard, 1973). If the animal is stimulated again at the primary site, it does not convulse on the first trial. A few trials are required to reach the $C C$ state, thus indicating negative transfer.

An interesting question is "At what point in a sequence of alternations will a rat's behavior become stable, i.e., show no positive or negative transfer effects?" In previous research in our laboratory (Gaito, Note 1), an interesting result was obtained. When rats were stimulated three times per day over 15 to 19 phases of sequential alternation (six CC within each phase), they tended to show an "oscillation effect" during these alternations, especially in the time between onset of stimulation and onset of convulsion (latency). They tended to low values with stimulation of the primary site and high values when the secondary site was being stimulated. Some rats persisted in this oscillation throughout the entire sequence of alternations. For example, a rat might show a mean latency of $5 \mathrm{sec}$ over the six trials of CC

I wish to acknowledge that Olive and Stephen Gaito constructed bipolar electrodes and that Paul Sanberg implanted electrodes in some of the rats. on the primary side. When stimulated in the secondary amygdala, the mean latency would be approximately $15 \mathrm{sec}$. This oscillation effect occurred in spite of a single phase of bilateral stimulation provided prior to unilateral stimulation with some rats and with two phases of bilateral stimulation introduced after the oscillation effect was pronounced with other rats. The effect was weaker in criterion data (i.e., number of trials to six CC) and almost nonexistent in the duration of convulsion data. In the present experiments, we were concerned with the effect of five phases of bilateral stimulation prior to first unilateral trials, and when the five phases were introduced after the oscillation effect was present.

\section{METHOD}

Twenty-six Wistar rats (approximately 150 days of age) were implanted bilaterally with bipolar electrodes in the amygdala. The brain coordinates for electrode implantation were the same as in many experiments in our laboratory (e.g., Gaito \& Gaito, 1974): $.5 \mathrm{~mm}$ posterior to bregma, $4.5 \mathrm{~mm}$ from midline, $8.5 \mathrm{~mm}$ from skull.

In Experiment 1, 14 rats were stimulated in one amygdala (side chosen randomly in each rat) for $30 \mathrm{sec}$ with a $60-\mathrm{Hz}$ sine wave of $100 \mu \mathrm{A}$ (peak to peak) using a Lafayette stimulator until six CC resulted. There were three trials per day, with $1 \mathrm{~h}$ between trials. On the day after the sixth CC, stimulation began at the secondary site and continued until six CC occurred. Then stimulation again at the primary site was instituted, etc. This sequential alternation of unilateral stimulation was continued for six or seven phases. Five phases of bilateral stimulation with the same intensity and duration then were provided for the nine rats which had oscillated, followed again by seven phases of sequential alternation. The first phase of this last stimulation series was with the side contralateral to that of the last phase in the first series of sequential alternations.

In Experiment 2, 12 rats were stimulated through five phases of bilateral stimulation, and then had seven phases of unilateral sequential alternations.

Some rats did not convulse within 30 trials during Phases 1 and 2 of the unilateral stimulations. Thus, the current intensity was increased slowly over trials, to a maximum of $560 \mu \mathrm{A}$ with several rats, until a $C C$ resulted. This intensity was maintained for the remaining phases. Because of our previous results, only the latency and criterion data were anlayzed in each experiment. 
Table 1

Number of Rats Showing Oscillation (O) or No Oscillation (NO) During Unilateral Stimulation (U)

\begin{tabular}{|c|c|c|c|c|c|c|}
\hline & \multicolumn{4}{|c|}{ Experiment 1 (U-B-U) } & \multirow{2}{*}{\multicolumn{2}{|c|}{$\begin{array}{c}\text { Experiment } 2 \\
(B-U)\end{array}$}} \\
\hline & \multicolumn{2}{|c|}{ First Series } & \multicolumn{2}{|c|}{ Second Series* } & & \\
\hline & $\mathrm{O}$ & $\mathbf{N}$ & $\mathrm{O}$ & $\mathbf{N}$ & $\mathrm{O}$ & $\mathrm{N}$ \\
\hline Latency & 8 & 6 & 6 & 3 & 10 & 2 \\
\hline Criterion & 7 & 7 & 3 & 6 & 7 & 5 \\
\hline
\end{tabular}

*Although only oscillators were run during the second series (9), the number of $N+O$ in the second series is greater than the number of $O$ in the first series; during the first series, some rats oscillated in both measures, whereas others oscillated only in one of the two.

\section{RESULTS}

The results for the two experiments are shown in Table 1. As in the previous work, the latency measure showed the stronger effect for oscillation, 8 of the 14 rats in Experiment 1 indicating oscillation during the first unilateral series. Three of the six rats not oscillating reached the CC stage on the second trial. It is probable that more trials prior to achieving a CC are required so as to establish the inhibitory tendency in one amygdala.

The five phases of bilateral stimulation disrupted the oscillation effect in the latency measure for three of the eight rats which had oscillated during the first unilateral series, and for four of the seven rats in the criterion measure. But five rats oscillated in both series of unilateral stimulations in the former data and three in the latter. Also, it is interesting to note that one latency nonoscillator in the first series began to oscillate during the second unilateral series. Thus, not only may bilateral stimulation act as a partial disrupter of the effect, but also it appears to set up conditions so as to precipitate oscillation. This aspect is shown clearly with Experiment 2, where 10 of 12 rats oscillated in the latency measure following five phases of bilateral stimulation.

With both groups, the criterion measure was less sensitive in indicating oscillation.

There are two types of oscillation observed in this experiment and in previous work (Gaito, Note 1). In one case, the low values appear for the side first stimulated (primary oscillation). On the other hand, the low values may appear for the second side stimulated (secondary oscillation). In the earlier experiments, primary oscillation predominated. In these experiments, primary oscillation also occurred most frequently. For Experiment 1, Series 1, the frequency for primary and secondary oscillation, respectively, was 5 and 3 for latency data and 5 and 2 for the criterion measure. In the second series, it was 4 and 2 for latency and 1 and 2 for criterion data. For Experiment 2, the values were 5 and 5 for latency and 6 and 1 for the criterion dependent variable.

Typical data for a rat from each experiment showing oscillation is provided in Table 2 . In one case, both latency and criterion aspects indicate primary oscillation for the seven phases following bilateral stimulation. In the second rat, secondary oscillation appears for both dependent variables only for four phases in both unilateral series. Thereafter, secondary oscillation occurs only in the latency measure.

Supplementing these quantitative data showing the "oscillation tendency" were qualitative behaviors. With some rats, when stimulating the side which showed high latency values, the rat would rear on hind paws and almost CC quickly, but appeared to be restrained for a few seconds before the CC ensued. For example, during unilateral phases, Rat 5 in Experiment 2 showed a CC immediately upon activating the stimulator for the primary site. When the secondary site was stimulated, this rat reared immediately but did not CC until approximately $10 \mathrm{sec}$ had elapsed. The rat appeared to be actively inhibited during this state of suspension prior to convulsing.

\section{DISCUSSION}

In Experiment 1. 9 of 14 rats oscillated; in Experiment 2. oscillation resulted with 10 of 12 rats. These results $(19$ oscillators with 26 rats) are consistent with the previous work (18 oscillators in 19 rats) and suggest that oscillation is a genuine phenomenon.

One might suggest that oscillation is a chance effect. However.

Table 2

Typical Oscillation Data for One Rat in Each Experiment

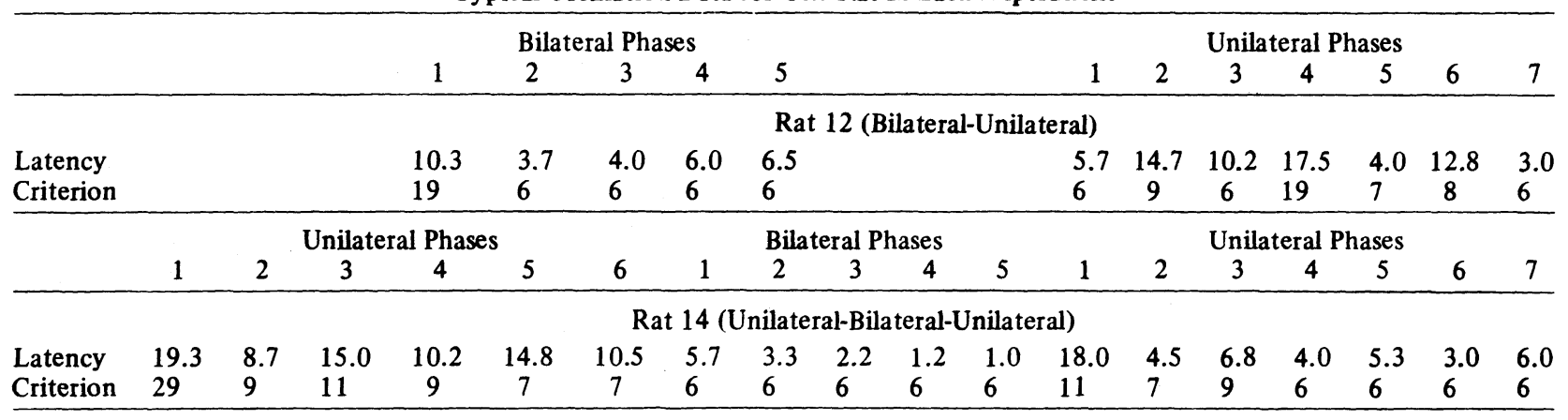


the likelihood of a number of rats showing a systematic change from low to high (or high to low) on successive phases is extremely low. Also one might state that oscillation is based on poor placement of one electrode in. or out of, the amygdala showing the high values. Although this latter explanation sounds like a reasonable one, there are a number of observations which make it appear unlikely.

(1) If poor placement is the basis for oscillation, the number of primary oscillators should be approximately equal to the number of secondary oscillators. However, there tend to be more primary oscillators than secondary oscillators. This difference was not pronounced in the present study, but in the previous research, 15 of 18 rats showing oscillation were primary oscillators.

(2) If this explanation is appropriate, oscillation should not occur after five phases of bilateral stimulation because such stimulation brings rats to quick attainment of the criterion, viz., in six trials for most rats, and to low values for latency. However, in Experiment 2. 10 of the 12 rats showed oscillation in latency data.

(3) Furthermore, oscillation should occur only over a few phases and then disappear, because the periodic stimulation lowers the threshold for seizure and both sides should become equally efficient (Tress \& Herberg, 1972). However, although there are a few rats who terminate oscillation after a few phases, most continue to oscillate throughout the entire series of phases, especially in the latency measure.

(4) Although the latency and duration measures go in the same direction in many cases (especially during the early phases), they are relatively independent on other occasions, as indicated in these experiments and in the previous research. Oscillation occurs more frequently in latency data than in the criterion measure. Poor electrode placement would be expected to affect the criterion measure, e.g., by requiring more trials to achieve the six CC. However, this effect need not be influencing the latency measure. In fact, in the previous research it was noted that the electrode did not have to be in the amygdala to achieve oscillation. A rat with a misplaced electrode (in the caudate putamen-globus pallidus area) provided primary oscillation in latency data. Even though $\mathbf{5 0}$ trials were required to reach criterion during stimulation of the primary site on the first phase (which is much more than usual), latencies were lower on this phase and on the third phase, showing excellent oscillation for the four phases on which this rat was run.

(5) Most rats begin oscillation on Phase 1. However, in a few cases, oscillation does not occur until Phase 3, e.g., Rat 6 of Experiment 2. Thus, by this phase, brain conditions should be sufficient to allow convulsions to ensue even though electrode placement may be slightly different for the two sides.

(6) The qualitative behavior discussed above might be another basis for rejecting the poor electrode placement interpretation for oscillation. Although a rat may rear quickly with stimulation of either side, the convulsion occurs immediately for one side but appears to be actively inhibited with stimulation of the other side. This appearance of active inhibition of potential behavior seems to argue against the notion that the longer time involved is due to poor electrode placement.
There appear to be two transfer processes occurring following unilateral stimulation to the seizure stage: one operating as a positive effect and the other as a negative one. The positive process probably is based on nerve pathway modification with resulting facilitation (Goddard, McIntyre, \& Leech, 1969). Such view is consistent with the electrophysiological changes which occur during the kindling event (Goddard, McIntyre, \& Leech, 1969; McIntyre \& Goddard, 1973). Presumably, this aspect is a long-term one which would remain relatively constant following a number of convulsions in both primary and secondary sites.

The second process is a negative one, an active inhibition effect. This process probably is responsible for the oscillation effect. The strength of the process would seem to vary with different stimulation conditions; possibly strength would increase with massing of trials, increasing number of convulsions (McIntyre \& Goddard, 1973), decreasing number of bilateral stimulation phases, and a number of other factors. This active inhibitory process may involve the same chemical which produces the interanimal retardation effect in the development of kindling in naive recipient rats (Gaito \& Gaito, 1974; Gaito, 1976).

The unilateral sequential alternation procedure appears to be useful for obtaining information on some aspects of underlying mechanisms via the oscillation effect. By determining variables which minimize or maximize this effect, one could gain clues concerning brain mechanisms which are responsible for the kindling effect.

\section{REFERENCE NOTE}

1. Gaito, J. An oscillation effect during sequential alternations of unilateral amygdaloid stimulations within the kindling paradigm. Submitted for publication, 1975.

\section{REFERENCES}

Garto, J. Pairing of the transfer experiment with the kindling paradigm: A summary of results. Bulletin of the Psychonomic Society, 1976, 7, 50-52.

Gaito, J., \& GaIto, S. T. Interanimal negative transfer of the kindling effect. Physiological Psychology, 1974, 2. 379-382.

Goddard, G. V., McIntYre, D. C., \& Leech, C. K. A permanent change in brain function resulting from daily electrical stimulation. Experimental Neurology, 1969. 25. 295-330.

MCINTYRE, D. C., \& GodDARD, G. V. Transfer, interference and spontaneous recovery of convulsions kindled from the rat amygdala. Electroencephalography and Clinical Neurophysiology, 1973, 35, 533-543.

Tress, K., \& Herberg, L. J. Permanent reduction in seizure threshold resulting from repeated electrical stimulation. Experimental Neurology, 1972, 37, 347-359.

(Received for publication December 1, 1975.) 